\title{
Arthroplasty implant biomaterial particle associated macrophages differentiate into lacunar bone resorbing cells
}

\author{
R Pandey, J Quinn, C Joyner, D W Murray, J T Triffitt, N A Athanasou
}

\begin{abstract}
Objective-To study the pathogenesis of aseptic loosening: in particular, to determine whether macrophages responding to particles of biomaterials commonly used in arthroplasty surgery for arthritis are capable of differentiating into osteoclastic bone resorbing cells, and the cellular and hormonal conditions required for this to occur.
\end{abstract}

Methods-Biomaterial particles (polymethylmethacrylate, high density polyethylene, titanium, chromium-cobalt, stainless steel) were implanted subcutaneously into mice. Macrophages were isolated from the foreign body granulomas that resulted, cultured on bone slices and coverslips, and assessed for both cytochemical and functional evidence of osteoclast differentiation.

Results-Tartrate resistant acid phosphatase (TRAP) negative macrophages isolated from granulomas containing particles of all types of biomaterial composition were capable of differentiating into TRAP positive cells capable of extensive lacunar bone resorption (assessed by scanning electron microscopy). The presence of both UMR106 rat osteoblast-like cells and 1,25-dihydroxy vitamin $D_{3}$ was necessary for this to occur.

Conclusion-All implant materials produce wear particles that are the focus of a heavy foreign body macrophage response in the fibrous membrane between a loose implant component and the host bone undergoing resorption. These findings underline the importance of biomaterial wear particle generation and the macrophage response to different types of biomaterial wear particles in the pathogenesis of aseptic loosening.

(Ann Rheum Dis 1996; 55: 388-395)

Although aseptic loosening of implanted prosthetic components is the commonest cause of failure of joint replacements, its pathogenesis remains unclear. In the fibrous membrane surrounding a loose cemented or uncemented implant, numerous macrophages and macrophage polykaryons are often found as part of the foreign body reaction to wear particles derived from various implant biomaterials. ${ }^{1-3}$ Considerable periprosthetic bone resorption is commonly seen around this mononuclear phagocyte rich membrane, but the precise role of macrophages and different biomaterial wear particles in causing this osteolysis is uncertain.

It has long been suspected that the cellular response to wear particles is an important factor in the osteolysis of aseptic loosening. Little or no macrophage infiltrate is seen in the membrane surrounding a well fixed prosthesis. $^{34}$ In contrast, numerous wear particles and a correspondingly heavy macrophage and macrophage polykaryon infiltrate are seen in cases of early loosening (aggressive granulomatosis) of both cemented and uncemented implants. ${ }^{5} 6$ Wear particle associated macrophages are known to produce prostaglandins and cytokines that can indirectly (via osteoblast stimulation) activate osteoclastic bone resorption. ${ }^{7}$ However, there is evidence that macrophages are also capable of resorbing bone directly. Macrophages can degrade devitalised bone particles, ${ }^{8}$ and both murine monocytes and various murine and human tissue macrophages, including alveolar and peritoneal macrophages, are known to differentiate in vitro into cells capable of osteoclast-like lacunar bone resorption under specific cellular and hormonal conditions. ${ }^{9-11}$ Quinn $e t$ al $^{12}$ found that murine inflammatory macrophages accumulating in response to polymethylmethacrylate (PMMA) particles were similarly capable of differentiating into osteoclast-like bone resorbing cells, indicating that the osteolysis of aseptic loosening could also result from this process.

In joint replacement surgery, biomaterial wear particles are derived from implant components composed of a number of different biomaterials including organic polymers, PMMA, and high density polyethylene (HDP), and metals of single element or metal alloy composition such as titanium, stainless steel, chromium-cobalt, and titanium alloy. A number of these biomaterial particles are known to show considerable variation in size and shape in situ, and these factors, in addition to their composition, are thought to influence the extent of the osteolysis that occurs in aseptic loosening. The aim of this study was to investigate further the role of macrophages associated with biomaterial wear particles in the osteolysis of aseptic loosening. Using a macrophage-bone stromal cell coculture model of pathological bone resorption,,$^{911} 12$ we 
investigated whether foreign body macrophages responding to particles of all the above biomaterials were capable of differentiating into osteoclastic bone resorbing cells. We also sought to assess whether particles of specific biomaterial composition strongly influenced this process.

\section{Materials and methods \\ BIOMATERIAL PARTICLES}

Particulates of titanium (Johnson Mathey, Herts, UK) (size $<80 \mu \mathrm{m}$ ) and stainless steel (size $10 \mu \mathrm{m}$ ) (Koch chemicals, UK) were obtained commercially from metal manufacturers. Particulates of chromium-cobalt $($ size $<40 \mu \mathrm{m})$ and HDP (size $<80 \mu \mathrm{m})$, were kindly provided by Corin, UK; these particles were derived from their implant component manufacturing process. PMMA particles (CMW Laboratories, UK) were prepared by crushing a block of acrylic cement using a mortar and pestle; they were then passed through a series of mesh sieves (Endecotts test sieve, Philip and Harris UK) until particles less than $80 \mu \mathrm{m}$ maximum size were obtained. The size range of the biomaterial particles used was confirmed by scanning electron microscopy. All biomaterial particles were sterilised by immersion in absolute alcohol in a large petri dish for 15 minutes. They were then dried and stored until required for use. HDP particles were placed in a $20 \mathrm{ml}$ container filled with absolute alcohol and required regular agitation to remain immersed. All biomaterial particles were then air dried in a laminar flow clean air hood before being implanted.

PREPARATION OF FOREIGN BODY GRANULOMAS IN MICE AND ISOLATION OF BIOMATERIAI

PARTICLE INDUCED MACROPHAGES

Separate gelatin capsules (size 2, Farillon Limited, Essex, UK) were loaded with $100 \mathrm{mg}$ of each of the above biomaterials particles and sterilised with ethylene dioxide. Each capsule was implanted subcutaneously into the dorsum of a six week old MF1 mouse (Harlan UK Ltd, Bicester, UK). Four mice were implanted with each biomaterial in each experiment. As negative control, mice were implanted with empty gelatin capsules. After six weeks, the mice were killed and the biomaterial particle induced granulomas removed under sterile conditions. Imprints of the cut surface of the granuloma were taken on glass slides for histochemical identification. One third of the lesion was fixed in formalin, routinely processed, and stained with haematoxylin and eosin or von Kossa stain for histological examination. The remainder of the lesion was curetted in $2 \mathrm{ml}$ of $\alpha$ minimal essential medium (MEM) (Sigma, UK) with added 10\% fetal calf serum (FCS) (Sigma), $10 \mathrm{IU} / \mathrm{ml}$ penicillin (Gibco, UK), $10 \mu \mathrm{g} / \mathrm{ml}$ streptomycin (Gibco) and $2 \mathrm{mmol} / \mathrm{l}$ glutamine (Gibco) (MEM/FCS). The tissue pieces were agitated with a smooth ended glass pipette to obtain a suspension of cells which was layered onto bone slices and coverslips as described below.
PREPARATION OF COCULTURES ON HUMAN

CORTICAL BONE SLICES AND COVERSLIPS

Human cortical bone slices (approximately $10 \mathrm{~mm}^{2}$ ), cut with a low speed bone saw using a diamond blade (Buehler Isomet IL) to produce a smooth surface that did not contain resorption lacunae, ${ }^{13}$ and $5 \mathrm{~mm}$ glass coverslips were placed in $6 \mathrm{~mm}$ wells of a 96 well tissue culture plate (Nunc, Denmark). UMR106 osteoblast-like cells (generously provided by Professor T J Martin, Melbourne, Australia), derived from a rat osteosarcoma cell line, ${ }^{14}$ were seeded onto coverslips and bone slices $\left(10^{5}\right.$ cells/well); these were cultured for 24 hours in $0.5 \mathrm{ml}$ MEM/FCS. The number of cells in the suspension isolated from each biomaterial granuloma was counted in a haemocytometer and the final concentration adjusted in MEM/FCS so that $5 \times 10^{3}$ cells (in 25-50 $\mu \mathrm{l}$ ) were added to each well containing a bone slice or coverslip. The cell suspension was settled onto bone slices (on average, 12 bone slices per biomaterial per experiment) for 60 minutes at $37^{\circ} \mathrm{C}$ in a $5 \%$ carbon dioxide incubator. The bone slices and coverslips were then removed from the $6 \mathrm{~mm}$ wells, washed vigorously in MEM/FCS to remove nonadherent cells, and placed in $16 \mathrm{~mm}$ wells of a 24 well tissue culture plate (Costar, USA) containing $1 \mathrm{ml} \mathrm{MEM} / \mathrm{FCS}$ with $2 \times 10^{-8} \mathrm{~mol} / 1$ of 1,25-dihydroxy vitamin $\mathrm{D}_{3}$ (Roche, UK). These cultures were maintained at $37^{\circ} \mathrm{C}$ in $5 \%$ carbon dioxide for up to 14 days. Culture medium containing 1,25-dihydroxy vitamin $\mathrm{D}_{3}$ was replenished every three days. After incubation, bone slices were removed from culture and processed for scanning electron microscopy as described below. A number of cells settled alone on glass coverslips were also challenged with salmon calcitonin (Rorer, UK) $1 \mathrm{ng} / \mathrm{ml}$ to observe if their activity or motility was inhibited.

As negative controls, the cell suspension obtained from each biomaterial granuloma was added to bone slices and coverslips that had not been previously seeded with UMR106 cells. These were cultured under conditions identical to those described above for 14 days. As a further negative control, UMR106 cells were cultured alone on bone slices for 14 days without addition of the cells isolated from the granulomas. In addition, PMMA and HDP granuloma derived macrophages were incubated alone in the presence and absence of 1,25-dihydroxy vitamin $\mathrm{D}_{3}$. The culture supernatant of UMR106 cells which were incubated on bone slices (both with and without granuloma derived cells) were also added to PMMA and HDP granuloma derived macrophages cultured for 14 days alone on bone slices.

HISTOCHEMICAL CHARACTERISATION OF BIOMATERIAL GRANULOMA DERIVED CELLS For histochemistry, cell preparations on coverslips were fixed in paraformaldehyde $2.5 \%$ in phosphate buffered saline (PBS) and stained for tartrate resistant acid phosphatase (TRAP) using naphthol AS-TR phosphate 
(Sigma) as a substrate, in the presence of 50 $\mathrm{mmol} / \mathrm{l}$ tartrate; the reaction product was stained with hexazotised pararosaniline (Sigma). ${ }^{15}$ For non-specific esterase staining, the cells cultured on coverslips were fixed in paraformaldehyde vapour and, using naphthylacetate (Sigma) as a substrate, the reaction product was coloured with fast blue salt (Sigma). ${ }^{16}$ The presence of F4/80 antigen on adherent macrophages was sought using an indirect immunoperoxidase technique as described previously. ${ }^{17}$ The total number of cells on each coverslip staining positive for F4/80 antibody was counted using the computerised image analysing software VIDAS 2.1 run on a Roche pathology work station (Kontron Elektronik, USA).

Cultures of UMR106 cells alone on glass coverslips and imprints from the cut surface of the biomaterial particle induced granulomas were similarly stained for TRAP and F4/80.

\section{SCANNING ELECTRON MICROSCOPE BONE}

RESORPTION ASSAY

Cell cultures on bone slices were removed from the wells after one, four, seven, and 14 days incubation. To remove the cells coating the surface of the bone slice, each bone slice was rinsed in PBS containing $0.2 \%$ EDTA then placed in $0.4 \%$ trypsin solution for 10 minutes. Bone slices were rinsed vigorously in distilled water and immersed in $0.25 \%$ ammonium hydroxide overnight; they were washed in distilled water, dehydrated in graded alcohols and air dried. The bone slices were mounted onto aluminium stubs using double sided sellotape, sputtered with gold, and examined using a Philips SEM 505 scanning electron microscope. The percentage of bone surface area resorbed on each bone slice was determined by tracing the outline of the pits from the screen of the scanning electron microscope and measuring the area using Plaqueman assay, an image processing computer program (Nuffield Department of Pathology, University of Oxford, UK).

STATISTICAL ANALYSIS

Each series of experiments was repeated at least four times. The number of cells derived from the various biomaterial granulomas which were added to each coverslip and the mean percentage of bone surface area resorbed are expressed as the mean (SEM). Significance was determined using Student's $t$ test.

\section{Results}

HISTOLOGY OF SUBCUTANEOUS GRANULOMAS FORMED IN RESPONSE TO BIOMATERIAL PARTICLES

On a gross scale, the subcutaneous biomaterial particle containing lesions were partly or completely surrounded by fibrous tissue that was thicker around implanted PMMA and HDP particles than around metallic particles. Histological examination of all the implanted lesions showed features of a foreign body granuloma in which there were numerous macrophages with plump eosinophilic cytoplasm and a single vesicular nucleus, and scattered macrophage polykaryons associated with the various biomaterial particles (fig 1A-C). Many macrophages and giant cells had phagocytosed polymer and metallic biomaterial particles. The heaviest macrophage and giant cell response was seen in granulomas formed in response to implanted PMMA and HDP particles. Calcified material was not seen histologically on either haematoxylin and eosin or von Kossa staining of any of the lesions, and granulomas were not formed in response to
Figure 1 Histology of foreign body granulomas showing numerous macrophages and occasional giant cells (open arrowheads) in response to subcutaneously implanted biomaterial particles (some arrowed $)$. (All $\times 400$.) A: Polymethylmethacrylate A: Polymethylmethacrylate polyethylene; $\boldsymbol{C}$ : titanium. D: Haematoxylin and eosin staining of mononuclear and multinucleated cells derived from imprint of cut surface of PMMA granuloma.
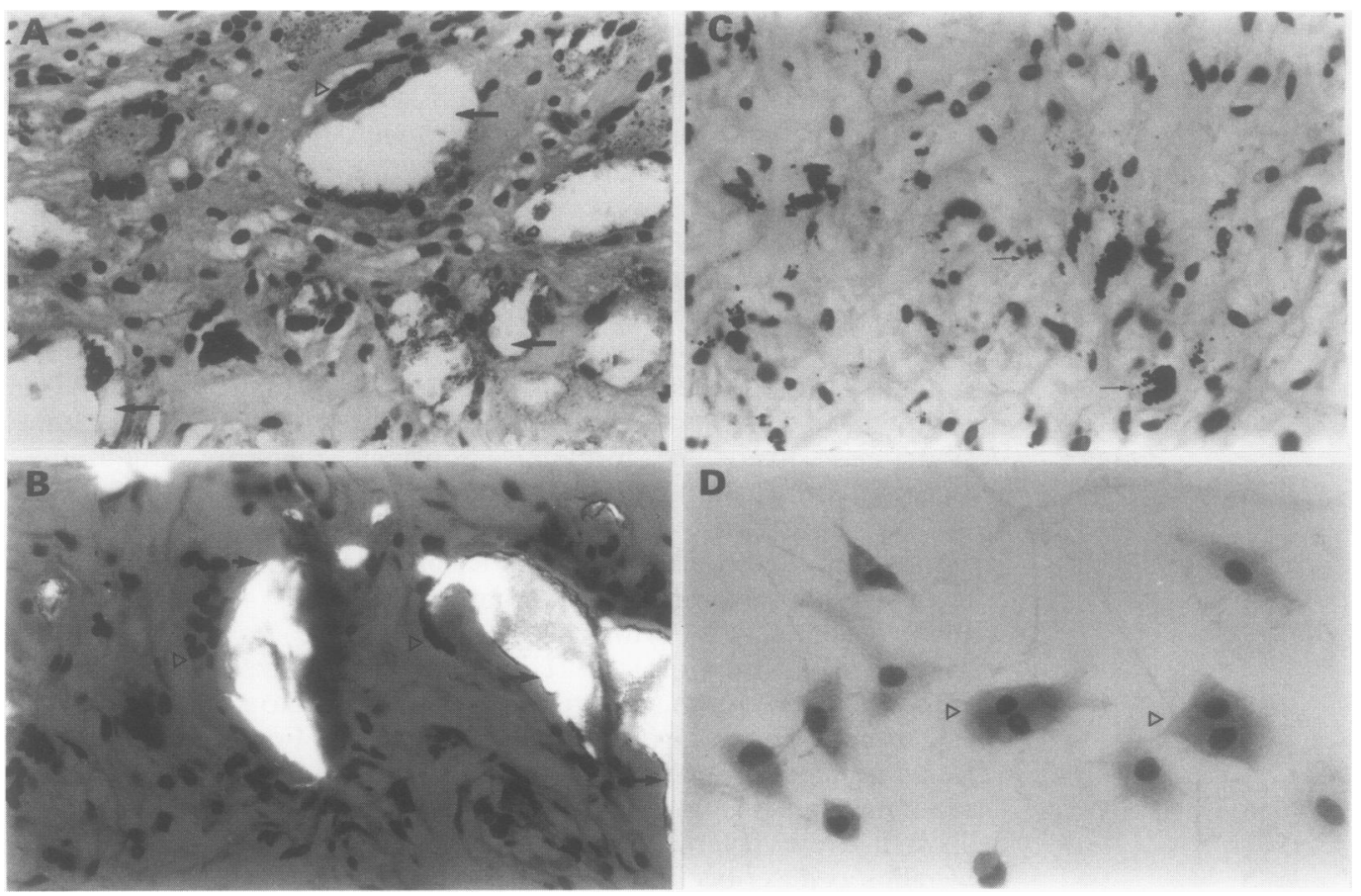


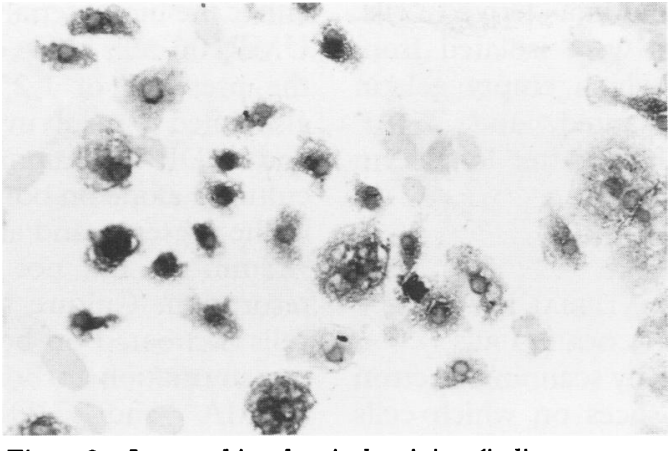

Figure 2 Immunohistochemical staining (indirect immunoperoxidase) of polymethylmethacrylate granuloma derived macrophages and UMR106 cells after seven days in coculture, showing numerous F4/80 positive cells (macrophages). ( $\times$ 400)

subcutaneous implantation of empty gelatin capsules. The imprints of the cut surface of the granulomas showed numerous mononuclear and multinucleated cells (fig 1D) that were non-specific esterase and $\mathrm{F} 4 / 80$ positive, but negative for the osteoclast associated enzyme TRAP.

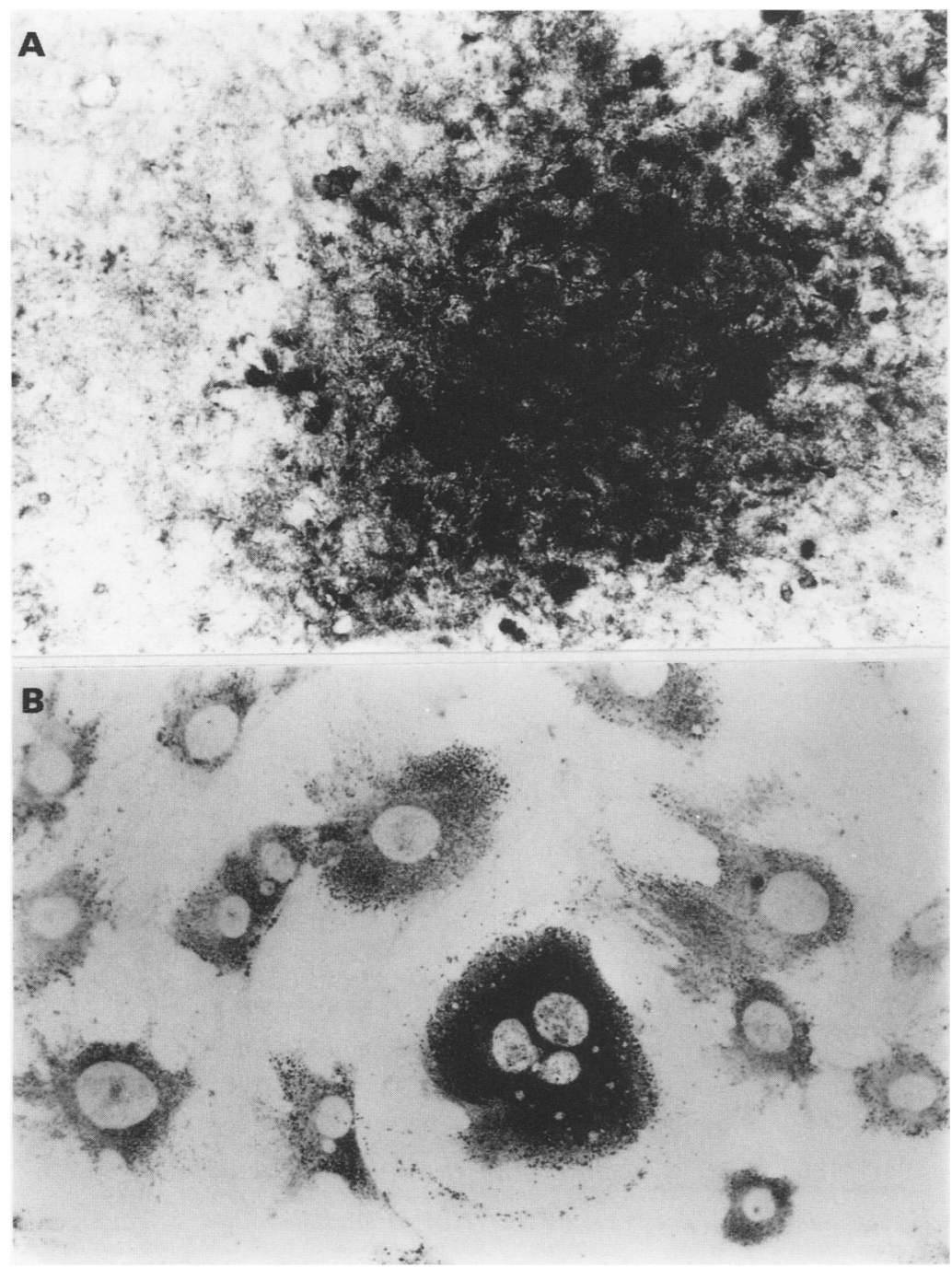

Figure 3 A: Cluster of tartrate resistant acid phosphatase (TRAP) positive cells present after seven days in coculture of high density polyethylene granuloma derived macrophages and UMR106 cells. (× 40.) B: High power view $(\times 400)$ from $A$, showing TRAP positive mononuclear and multinucleated cells.
CHARACTERISATION OF CELLS ISOLATED FROM THE GRANULOMAS

Adherent cells isolated from all the granulomas formed in response to implanted biomaterial particles were settled alone on coverslips for 24 hours and identified as macrophages on the basis that they were $(>90 \%)$ non-specific esterase and $F 4 / 80$ positive, but entirely negative for TRAP. These isolated cells, when cultured on coverslips alone, showed no morphological response to calcitonin. After seven days of coculture of UMR106 cells in the presence of 1,25-dihydroxy vitamin $D_{3}$, coverslips contained large numbers of nonspecific esterase and $\mathrm{F} 4 / 80$ positive cells (fig 2) in addition to scattered clusters of TRAP positive cells (fig 3). TRAP positive cells were not seen in seven and 14 day cultures of UMR106 cells alone (either in the presence or absence of 1,25-dihydroxy vitamin $\mathrm{D}_{3}$ ), nor were they found in macrophage cultures to which UMR106 cells or 1,25-dihydroxy vitamin $\mathrm{D}_{3}$ had not been added.

Abundant macrophages were isolated from all the various biomaterial particle induced granulomas. Although the same number of granuloma derived cells for each biomaterial was settled initially on each coverslip, it was noted that after seven days incubation the number of $F 4 / 80$ positive cells in PMMA granuloma derived cocultures was significantly greater than in those containing titanium, stainless steel, and HDP derived cells (fig 4). In addition, significantly fewer $\mathrm{F} 4 / 80$ positive cells were found in chromium-cobalt granuloma derived cells. After seven days incubation, staining for TRAP was most marked in cocultures containing PMMA,

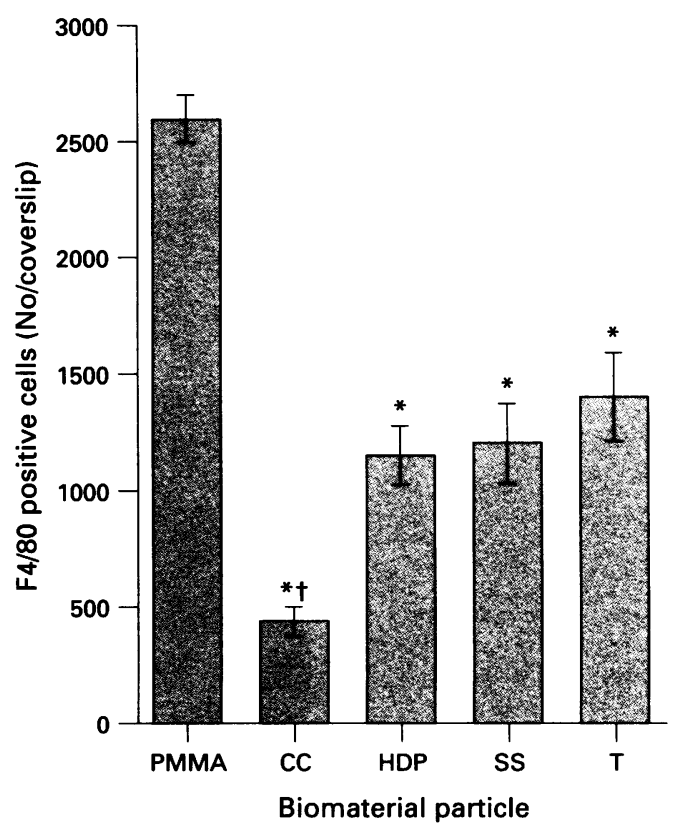

Figure 4 Mean (SEM) number of F4/80 positive cells (macrophages) derived from each biomaterial granuloma (polymethylmethacrylate (PMMA), chromium-cobalt (CC), high density polyethylene (HDP), stainless steel (SS), or titanium (T) found on coverslips after seven days in coculture with UMR106 cells. Statistically significant differences $(p<0.01)$ : *ompared with PMMA granulomas; fcompared with HDP, titanium, and stainless steel granulomas. 
HDP, and titanium granuloma derived cells. Few F4/80 positive cells were isolated from subcutaneous tissue in which empty gelatin capsules had been implanted, and TRAP positive cells were not found after long term coculture with UMR106 cells.

BONE RESORPTION BY BIOMATERIAL PARTICLE DERIVED MACROPHAGES IN COCULTURES

Resorption pits were seen by scanning electron microscopy upon bone slices on which cells isolated from all biomaterial granulomas were cocultured with UMR106 cells in the presence of 1,25-dihydroxy vitamin $\mathrm{D}_{3}$. Resorption pits were first evident after four days coculture on bone slices. The resorption pits were similar in size and morphology to those known to be formed by osteoclasts, ${ }^{18}$ but were far more numerous and generally larger than those formed by osteoclasts isolated directly from rodent bones. They ranged from individual small circular resorption pits (approximately 5 $\mu \mathrm{m}$ diameter) to large (often greater than 500 $\mu \mathrm{m}$ diameter) confluent areas of resorption composed of numerous pits (fig 5). Resorption pits were not found on bone slices on which UMR106 cells were cultured with a cell suspension isolated from negative controlsthat is, where a gelatin capsule alone (not containing biomaterial particles) was implanted subcutaneously. Resorption pits were not found on bone slices after coculture of UMR106 cells and granuloma derived macrophages for only one day. Culture of
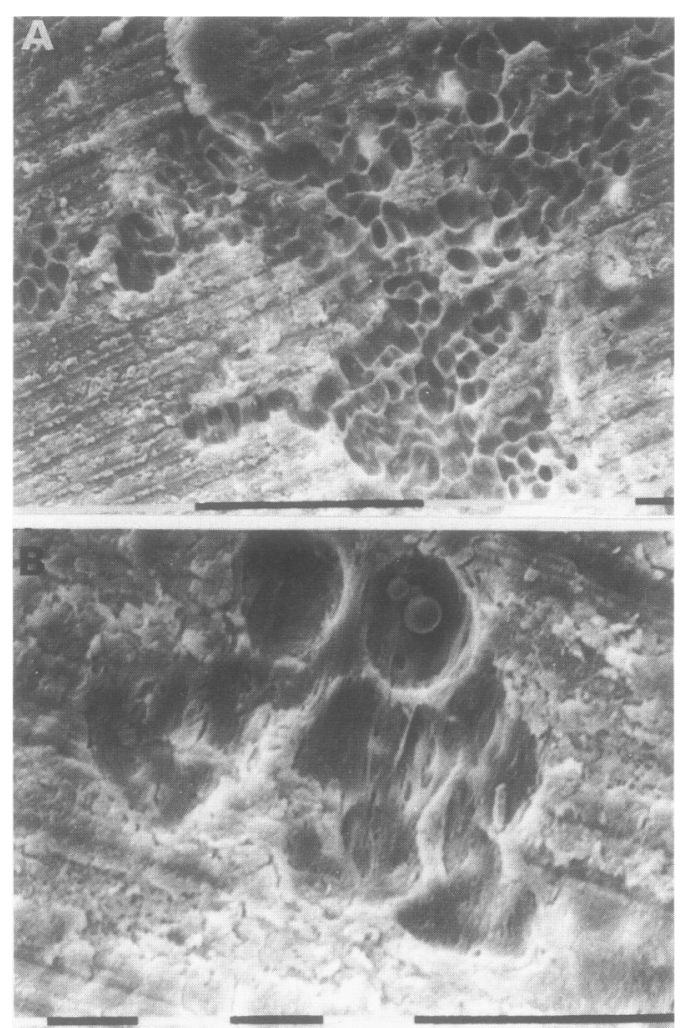

Figure 5 Scanning electron micrographs showing extensive resorption pit formation on bone slices on which UMR106 cells were cocultured for 14 days with foreign body macrophages derived from granulomas containing biomaterial paricles. A: Polymethylmethad (long black bar $=100 \mu \mathrm{m}$ ). B: Titanium (short black bar $=10 \mu \mathrm{m}$ ) either the biomaterial induced macrophages or UMR 106 cells alone on cortical bone slices, in the presence of 1,25-dihydroxy vitamin $\mathrm{D}_{3}$, also failed to result in bone resorption. PMMA and HDP granuloma derived macrophages cultured alone on bone slices for up to 14 days in the presence and absence of 1,25-dihydroxy vitamin $\mathrm{D}_{3}$ did not show evidence of bone resorption. Culture supernatant of UMR106 cells incubated on bone slices did not induce transformation to bone resorbing cells of the PMMA and HDP granuloma derived macrophages that had been incubated alone on bone slices.

Cocultures containing PMMA granuloma derived macrophages showed the greatest percentage of surface area of bone resorbed per bone slice, with cocultures containing HDP, titanium, chromium-cobalt, and stainless steel associated macrophages showing, respectively, successively less resorption (fig 6). Resorption associated with PMMA granuloma derived macrophages was significantly greater than that of macrophages associated with HDP $(p<0.05)$, titanium $(p<0.05)$, chromiumcobalt $(p<0.01)$, and stainless steel granulomas $(p<0.01)$. Resorption associated with HDP and titanium granuloma derived macrophages was also significantly greater than that of macrophages derived from chromiumcobalt and stainless steel granulomas (all $\mathrm{p}<0.05)$. No significant difference was seen in the amount of macrophage associated bone resorption between cultures of HDP and titanium or chromium-cobalt and stainless steel granuloma derived cells. The maximum number of large resorption areas (which often

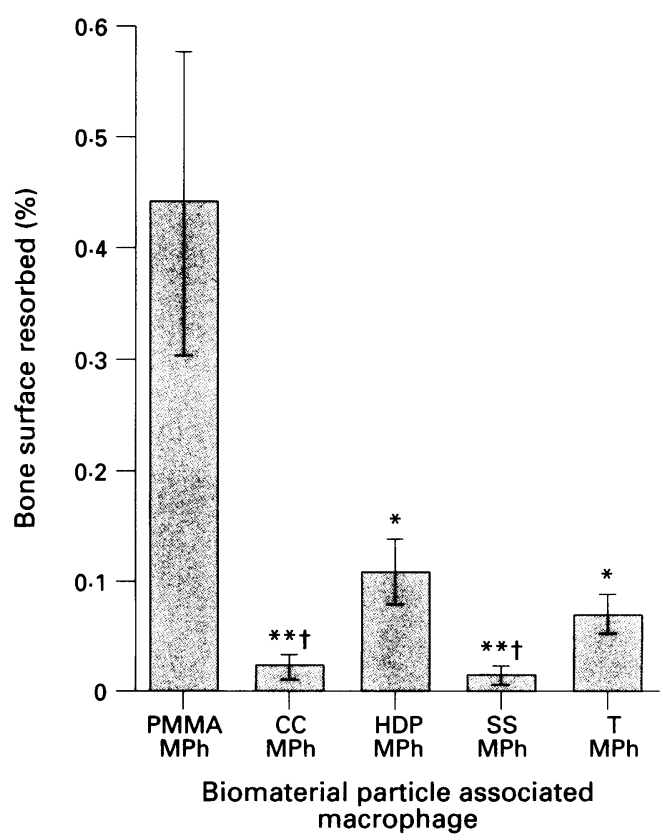

Figure 6 Mean percentage of total bone surface area resorbed by the various types of biomaterial granuloma derived macrophages (polymethylmethacrylate (PMMA $M P h)$, chromium-cobalt (CC MPh), high density $M P h)$, chromium-cobalt (CC MPh), high density
polyethylene (HDP MPh), stainless steel (SS MPh), or titanium (T MPh)) cocultured with UMR106 cells for 14 days. Statistically significant differences: $p<0.05$ compared with *bone resorption by PMMA associated macrophages, or tbone resorption by HDP and titanium associated macrophages; ${ }^{\star} p<0.01$ compared with bone resorption by PMMA associated macrophages. 
comprised several hundred individual resorption pits, each representing a separate single episode of lacunar bone resorption) seen on a single bone slice was 150 . The maximum percentage surface area of a bone slice resorbed was $2.5 \%$ (that is, $250000 \mu \mathrm{m}^{2}$ : approximately 10000 individual resorption pits). Both maximum values were seen on a bone slice upon which $5 \times 10^{3}$ PMMA granuloma derived cells had been settled and cocultured with UMR106 cells for 14 days.

\section{Discussion}

A heavy macrophage and macrophage polykaryon infiltrate is commonly seen in response to the accumulation of biomaterial wear particle in the arthroplasty tissues surrounding a loose cemented or uncemented prosthesis. ${ }^{1-3}$ A correlation exists between the amount of wear debris and the extent of this mononuclear phagocyte response; this, in turn, is reflected clinically in the severity and rapidity of onset of aseptic loosening. ${ }^{6}{ }^{19}$ The results of this study emphasise the importance of the macrophage response to wear particles in the pathogenesis of aseptic loosening. Foreign body macrophages aggregating in response to particles of various polymer and metal biomaterials commonly used in joint replacements were all shown to be capable of differentiating into osteoclast-like cells capable of extensive bone resorption. In addition, macrophages reacting to particular biomaterial particles (notably PMMA associated macrophages), were associated with significantly more resorption, suggesting that specific biomaterial factors may have a role in influencing this mechanism.

The subcutaneous foreign body granulomas formed in response to the various implanted biomaterial particles contained a macrophage and macrophage polykaryon infiltrate similar to that found in the fibrous tissue membrane around arthroplasty components. As in the arthroplasty membrane in situ, larger biomaterial particles were either engulfed or surrounded by macrophages and macrophage polykaryons, whereas smaller particles were phagocytosed by these cells. However, irrespective of the type of biomaterial implanted, the mononuclear phagocytes elicited in this foreign body response were all capable of differentiating into cells capable of extensive bone resorption. Thus wear particles shed from all biomaterials commonly used in joint replacement surgery must be regarded as having the potential to form the focus of a macrophage response from which aseptic loosening can result. The extent of the lacunar resorption seen in our cocultures can be appreciated when the maximum surface area of resorbed bone-that is, $250000 \mu^{2}$, which represents approximately 10000 individual resorption pits-is compared with that produced by isolated rodent osteoclasts which, even in the presence of UMR106 cells and 1,25-dihydroxy vitamin $D_{3}$ (conditions of maximal stimulation), produce fewer than 60 small pits; ${ }^{20}$ this was noted on a bone slice containing PMMA granuloma derived macrophages $\left(5 \times 10^{3}\right.$ cells per well). In terms of the number of pits and surface area, the amount of lacunar bone resorption seen in this coculture system is similar to that of mouse bone marrow haemopoietic cell cultures, ${ }^{21}$ in which $10^{6}$ cells are routinely added to each bone slice.

Cells isolated from biomaterial granulomas were initially F4/80 and non-specific esterase positive, calcitonin response and TRAP negative, and did not form resorption pits when cultured alone on bone slices-features in keeping with their macrophage phenotype; the absence of characteristic osteoclastic cytochemical and functional features would argue against these cells being fully differentiated osteoclast precursors. However, after seven days in coculture with UMR106 osteoblast-like stromal cells in the presence of 1,25-dihydroxy vitamin $\mathrm{D}_{3}$, isolated cells became TRAP positive in a manner similar to that reported for both mouse bone marrow cultures $^{22}$ and cocultures of monocytes and bone derived stromal cells, ${ }^{9}{ }^{23}$ all of which result in generation of TRAP positive, calcitonin receptor positive cells capable of lacunar bone resorption; this, in itself, is evidence of a change in phenotype consistent with osteoclastic differentiation, but cannot be said to be a specific effect, as TRAP can be expressed by some resident (for example alveolar) and highly activated macrophages. ${ }^{24}$ However, quite specific for osteoclast differentiation is the fact that extensive lacunar resorption pit formation was associated with the appearance of TRAP positive cells in these cocultures. The failure of previous studies to show that biomaterial wear particle associated macrophages can directly resorb bone is most probably attributable to the fact that the two essential elements required for macrophageosteoclast differentiation (1,25-dihydroxy vitamin $\mathrm{D}_{3}$ and a bone derived stromal cell population) were not included in macrophage cultures on bone slices. ${ }^{25}$ Contact with a specific type of bone stromal cell appears to be necessary for the formation of osteoclastlike cells from a mononuclear phagocyte population. ${ }^{9-12} 23$ In this study, macrophages were cocultured with UMR106 rat osteosarcoma cells, which have a well characterised osteoblast phenotype. ${ }^{14}$ The osteolysis of aseptic loosening occurs at the bone-implant interface, where biomaterial wear particle associated macrophages would come in contact with cells of the osteoblastic lineage.

A small proportion of DNA synthesising mononuclear phagocytes are known to form part of the macrophage pool in various tissues. ${ }^{26}$ These cells cannot be distinguished morphologically from mature tissue macrophages but, unlike them, are capable of proliferation. As mitotic proliferation persisting for the duration of the lesion is known to occur in the mononuclear phagocyte population of inflammatory granulomas, ${ }^{27}{ }^{28}$ it is possible that the heavy foreign body macrophage infiltrate of the various biomaterial granulomas includes a large 
number of immature mononuclear phagocytes that are capable of further proliferation. Under specific conditions, such immature cells may also be capable of further site dependent differentiation to highly specialised cells of the mononuclear phagocyte system; in bone this would be manifested as contact with a bone stromal cell population and exposure to 1,25-dihydroxy vitamin $\mathrm{D}_{3}$, resulting in differentiation towards the specialised cell of the mononuclear phagocyte system appropriate to bone-that is, the osteoclast.

Although our results show that osteolysis is a product of the macrophage response to biomaterial particles per se rather than to a particular biomaterial type, more bone resorption was seen in cocultures containing macrophages derived from granulomas containing particular organic polymer (PMMA and HDP) biomaterials than in those containing metal (titanium) biomaterials. This finding suggests that particular biomaterial factors may influence the differentiation or function of these bone resorbing cells. It was notable that the subcutaneous granulomas containing PMMA, HDP, and titanium particles were all associated with a heavy macrophage and macrophage polykaryon foreign body response. Macrophages within such inflammatory granulomas are known not only to be capable of proliferation, but also to be highly activated-that is, to be in an enhanced state of competence to mediate or complete a complex function. ${ }^{28}$ Thus it is possible that larger numbers of activated macrophages capable of osteoclastic differentiation are present in the heavier macrophage infiltrate associated with these particular biomaterial granulomas. Moreover, though the same number of macrophages from each biomaterial granuloma was settled on each bone slice, immunocytochemical staining for F4/80 (a macrophage associated antigen) showed that, after seven days, cultures of cells isolated from PMMA, HDP, and titanium granulomas contained more macrophages than those derived from others in which little resorption was seen, such as chromium-cobalt granulomas. Whether this reflects a toxic effect by chromium-cobalt on macrophage survival, differentiation, or function is uncertain, but there is some evidence to suggest that this could be a factor. ${ }^{29}$ Macrophage number and biomaterial type, however, do not appear to be the only criteria determining the amount of bone resorption resulting from this process, as demonstrated by our findings with stainless steel particles, with which, despite large numbers of macrophages being isolated from the granulomas, relatively little lacunar resorption was noted following coculture. Differences in the stromal cell response to different types of metallic wear particles in vitro, in terms of enzyme induction and cell proliferation, could also possibly account for some of the differences in the extent of the osteolysis. ${ }^{30}$ In addition there are, apart from biomaterial composition, numerous other biomaterial factors such as particle size, shape, surface regularity, and surface area, that are known to influence a number of macrophage responses, including bone resorption. ${ }^{31} 32$ Particle size, in particular, is believed to be an important determinant of the macrophage response associated with aseptic loosening; ${ }^{733}$ as the particle sizes of each of the biomaterials studied were not identical, this could certainly have influenced the amount of resorption by cells isolated from the various biomaterial granulomas in this study.

Even allowing for variation in the size of implanted PMMA particles (all of which were less than $80 \mu \mathrm{m}$ ), it was notable that macrophages derived from PMMA granulomas produced considerably more resorption than macrophages associated with other biomaterials. Wear particles of PMMA evoke a heavy foreign body macrophage response in the fibrous membrane surrounding cemented arthroplasty components; ${ }^{2334}$ this response has been consistently associated with aseptic loosening and has led to the coining of the term 'cement disease' for this phenomenon. ${ }^{34}$ Clinically, considerable localised osteolysis has also been associated with the presence of abundant PMMA wear particles alone. ${ }^{35}$ More recently, it has been shown that bulk PMMA does not evoke the heavy macrophage response associated with particulate forms of PMMA. ${ }^{36}$

In aseptic loosening, the shedding of biomaterial wear particles appears to be central to the pathogenesis of osteolysis, as it is their presence that elicits a macrophage response. Biomaterial wear particle associated macrophages produce numerous local humoral factors, such as cytokines, prostaglandins, and growth factors, that are capable of stimulating the osteoclastic bone resorption which contributes to aseptic loosening. ${ }^{7} 323738$ These factors are also known to influence macrophage and osteoclast differentiation ${ }^{23} 39$ and thus would be likely to influence the differentiation of biomaterial associated macrophages into bone resorbing cells. The in vitro model of osteolysis caused by biomaterial associated macrophages that has been outlined in this study should provide a means whereby the effect of these hormonal factors (in addition to the effects of specific biomaterial and cellular factors) on this process can be quantifiably determined in terms of the main outcome in aseptic loosening - that is, bone resorption.

This work was supported by the British Orthopaedic Association Wishbone Trust and the Wellcome Trust. Mr Pandey was supported by the Lord Nuffield Charitable Orthopaedic Trust. We thank Mr R de Steiger for helping us to obtain some of the particles and Mrs L Akers for typing the
manuscript.

1 Revell P A. Tissue reactions to joint prostheses and the products of wear and corrosion. In: Berry C, ed. Current trends in pathology. Berlin: Springer-Verlag, 1982; 73-101.

2 Lennox D W, Schofield B W, McDonald D F, Riley L H. A histological comparison of aseptic loosening of cemented, press-fit, and biologic ingrowth prostheses. Clin Orthop 1987; 225: 171-91.

3 Bullough P G, DiCarlo E F, Hansraj K K, Neves M C. Pathologic studies of total joint replacement. Orthop Clin North Am 1988; 19: 611-25.

4 Malcolm A J. Pathology of longstanding cemented total hip replacement in Charnley's cases. F Bone foint Surg Br 1988; 70: 153 . 
5 Santavirta S, Hoikka V, Eskola A, Kontinnen Y, Paavilainen $\mathrm{T}$, Tallroth V. Aggressive granulomatous lesions in cementless total hip arthroplasty. $\mathcal{F}$ Bone foint Surg $\mathrm{Br}$ 1990; 72: $980-4$.

6 Santavirta S, Konttinen T Y, Bergroth V, Eskola A, Tallroth K, Lindholm T S. Aggressive granulomatous lesions associated with hip arthroplasty: immunopathological studies. $\mathcal{f}$ Bone foint Surg Am 1990; 72: patholo

7 Amstutz H C, Campbell P, Kossovsky N, Clarke I C. Mechanism and clinical significance of wear debrisinduced osteolysis. Clin Orthop 1992; 276: 7-18.

8 Teitelbaum S L, Stewart C C, Kahn A J. Rodent peritoneal macrophages as bone resorbing cells. Calcif Tissue Int 1979; 27: 255-61.

9 Udagawa N, Takahashi N, Akatsu T, et al. Origin of osteoclasts: mature monocytes and macrophages are capable of differentiating into osteoclasts under a suitable microenvironment prepared by bone marrow-derived stromal cells. Proc Natl Acad Sci USA 1990; 87: 7260-4.

10 Athanasou N A, Quinn J. Human tumour-associated macrophages are capable of bone resorption. Br $\mathcal{F}$ Cancer 1992; 65: 523-6.

11 Quinn J M, Sabokbar A S, Athanasou N A. Cells of the mononuclear phagocyte series differentiate into osteoclastic lacunar bone resorbing cells. F Pathol 1996 In press.

12 Quinn J M, Joyner C, Triffitt J T, Athanasou N A. Polymethylmethacrylate-induced inflammatory macrophages resorb bone. $f$ Bone foint Surg $\mathrm{Br}$ 1992; 74: 652-8.

13 Athanasou N A, Wells C A, Quinn J. The origin and nature of stromal osteoclast-like multinucleated giant cells in breast carcinoma: implications for tumour osteolysis and macrophage biology. Br f Cancer 1989; 59: 491-8.

14 Partridge N, Alcorn D, Michelangeli V, Kemp E, Ryan G, Martin T J. Functional properties of hormonal responsive cultured normal and malignant rat osteoblastic cells. Endocrinology 1981; 108: 213-9.

15 Minkin C. Bone acid phosphatase: tartrate-resistant acid phosphatase as a marker of osteoclast function. Calcif phosphatase as a marker of
Tissue Int 1982; 34: 285-90.

16 Kaplow L S. Cytochemical identification of mononuclear macrophages. In: Herscowitz $\mathrm{H}$ B, Holden $\mathrm{H}$ T, Bellanti J A, Ghaffer A, eds. Manual of macrophage methodology collection, characterization and function. New York: Marcel Dekker Inc, 1981; 199-207.

17 Austyn J M, Gordon S. F4/80, a monoclonal antibody directed specifically against the mouse macrophage. Eur f Immunol 1981; 11: 805-15.

18 Chambers T J, Revell P A, Fuller K, Athanasou N A. Resorption of bone by isolated rabbit osteoclasts. $\mathcal{f}$ Cell Sci 1984; 66: 383-99.

19 Harris W H, Schiller A L, Scholler J-M, Freiberg R A, Scott R. Extensive localised bone resorption in the femur following total hip replacement. $\mathcal{f}$ Bone foint Surg $\mathrm{Am}$ following total hip

20 McSheehy P M J, Chambers T J. 1,25 dihydroxyvitamin $D_{3}$ stimulates rat osteoblastic cells to release a soluble factor that increases osteoclastic bone resorption. 7 Clin Invest 1987; 80: 425-9.
21 Hattersley G, Chambers T J. Calcitonin receptors as markers for osteoclastic differentiation: correlation between generation of bone-resorptive cells and cells that express calcitonin receptors in mouse bone marrow cultures. Endocrinology 1989; 125: 1606-12.

22 Takahashi N, Yamana $\mathrm{H}$, Yoshiki S, et al. Osteoclast-like cell formation and its regulation by osteotropic hormones in mouse bone marrow cultures. Endocrinology 1988; 122: mouse bone

23 Quinn J M W, McGee J O'D, Athanasou N A. Cellular and hormonal factors influencing monocyte differentiation into osteoclastic bone-resorbing cells. Endocrinology 1994; 134: 2416-23.

24 Andersson G, Ek-Rylander B, Minkin C. Acid phosphatases. In: Rifkin BR, Gay CV, eds. Biology and physiology of the osteoclast. Boca Raton: CRC Press, 1992; 55-80.

25 Pazzaglia U E, Pringle J A S. The role of macrophages and giant cells-loosening in joint replacement. Acta Orthop Trauma Surg 1988; 107: 20-6.

26 van Furth $R$. Origin and turnover of monocytes and macrophages. Curr Top Pathol 1989; 79: 125-50.

27 Spector W, Lykke A W J. The cellular evolution of inflammatory granulomata. F Pathol 1966; 92: 163-72.

28 Adams D O, Hamilton T A. The cell biology of macrophage activation. Ann Rev Immunol 1984; 2: 283-330.

29 Haynes D R, Rogers S D, Hay S, Pearcy M J, Howie D W. The difference in toxicity and release of bone resorbing mediators induced by titanium and cobalt-chromium alloy wear particles. F Bone foint Surg Am 1993; 75: 825-33.

30 Maloney W J, Lane Smith R, Castro F, Schormann D. Fibroblast response to metallic debris in vitro. $\mathcal{F}$ Bone foint Surg Am 1993; 75: 835-45.

31 Gelb H, Schumacher H R, Cuckler J, Baker D G. In vivo inflammatory response to polymethylmethacrylate debris: effect of size, morphology and surface area. 7 Orthop Res 1994; 12: 83-92.

32 Glant T T, Jacobs J J, Molnar G, Shanbhag A S, Valyon M, Galante J O. Bone resorption activity of particulate-stimulated macrophages. $¥$ Bone Miner Res particulate-stimulated

33 Johanson N A, Bullough P G, Wilson P D, Salvati E A, Ranawat C S. The microscopic anatomy of the bonecement interface in failed total hip arthroplasties. Clin Orthop 1987; 218: 123-35.

34 Jones L C, Hungerford D S. Cement disease. Clin Orthop 1987; 225: 192-206.

35 Jasty M J, Floyd W E, Schiller A L, Goldring S R Harris W H. Localised osteolysis in stable non-septic total hip replacement. F Bone foint Surg Am 1986; 68: 912-9.

36 Jasty M, Jiranek W, Harris W H. Acrylic fragmentation in total hip replacements and its biological consequences. Clin Orthop 1992; 285: 116-28.

37 Murray D W, Rushton N. Macrophages stimulate bone resorption when they phagocytose particles. $\mathcal{F}$ Bone foint resorption when they phagoc

38 Jiranek W A, Machado M, Jasty M, et al. Production of cytokines around loosened cemented acetabular components. F Bone foint Surg Am 1993; 75: 863-79.

39 Suda T, Takahashi N, Martin T J. Modulation of osteoclast differentiation. Endocr Rev 1992; 13: 66-80. 